\title{
Quantum optics experiments with single photons for undergraduate laboratories
}

Enrique Galvez, Mark Beck

Enrique J. Galvez, Mark Beck, "Quantum optics experiments with single photons for undergraduate laboratories," Proc. SPIE 9665, Tenth International Topical Meeting on Education and Training in Optics and Photonics, 966513 (3 June 2007); doi: 10.1117/12.2207351

SPIE Event: Tenth International Topical Meeting on Education and Training in Optics and Photonics, 2007, Ottawa, Ontario, Canada 


\title{
Quantum Optics Experiments with Single Photons for Undergraduate Laboratories
}

\author{
Enrique J. Galvez \\ Department of Physics and Astronomy, Colgate University, Hamilton, NY 13346, USA, \\ (315) 228-7205,(315) 228-7205 (fax), egalvez@mail.colgate.edu \\ Mark Beck \\ Department. of Physics, Whitman College, Walla Walla, WA 99362, USA \\ (509) 527-5260, (509) 527-5260 (fax), beckmk@whitman.edu
}

\begin{abstract}
We present new results of interference experiments for undergraduates that underscore the quantum nature of the light. The experiments use parametric down-conversion to generate pairs of correlated photons. The experiments involve one- and two-photon interference schemes that rely on first and second-order coherence effects. They can be used as a complement to teaching quantum mechanics or quantum optics.

(C)2007 Optical Society of America

OCIS codes: (000.2060) Education, (270.0270) Quantum optics.
\end{abstract}

\section{Introduction}

Developments in the technology for producing and detecting correlated photon pairs via parametric down conversion have enabled the implementation of undergraduate-level laboratories for demonstrating fundamental quantum mechanical principles, such as superposition and entanglement. Whitman College and Colgate University have put much effort in developing undergraduate laboratories with correlated photons [1-5], and have recently started offering undergraduate quantum mechanics courses with associated laboratories based on these types of experiments.

Laboratories with correlated photons are important because they underscore fundamental concepts of quantum mechanics. They allow students to learn quantum mechanics via experimentation and thus start their quantum physics education from a position where they can gain valuable physical intuition. Experiments on interference of light at the single-photon limit serve as exercises in quantum mechanical concepts and algebra. Thus they constitute direct applications of a topic that is otherwise purely theoretical and abstract. An interesting feature of these types of experiments is that they give the instructor the flexibility to tailor the explanation of the results to his or her quantum mechanical formalism.

We use an increasingly popular source of correlated photons: spontaneous parametric down conversion. It consists of sending a pump laser beam to a nonlinear crystal to produce photon pairs that are correlated in time, energy, momentum and polarization. The pairs can be used as a source of non-classical light. In some cases one photon of a pair heralds the other one going through an interferometer, and in other cases both photons go through the interferometer for demonstrating richer quantum mechanical effects. Many experiments with correlated photons, in particular the ones presented in this article, cannot be reproduced by an attenuated source of light. With special modifications the source can produce photon pairs entangled in polarization, and thus enabling tests of Bell's inequalities $[3,6,7]$.

In previous publications we presented interference experiments for use in undergraduate laboratories $[1,2,5]$. In this article we expand upon some of those reports with the results of more recent work. In Sec. 2 we present an experiment that combines single-photon interference in a polarization interferometer with the "single-photon test." The latter is a second order coherence correlation test that reveals, and even proves, the quantum nature of the photon. In Sec. 3 we follow with a two-photon interference experiment where we study the photon correlations coming off the two ports of a Mach-Zehnder interferometer.

Tenth International Topical Meeting on Education and Training in Optics and Photonics, edited by Marc Nantel, Proc. of SPIE Vol. 9665, 966513 - (C) 2007 SPIE, OSA, IEEE, ICO doi: $10.1117 / 12.2207351$ 


\section{Single-photon interference}

In Ref. [1] we describe an undergraduate experiment that demonstrates that light is made of photons. By this we mean an experiment that requires a quantum mechanical treatment of the field for an explanation (i.e., an experiment that cannot be explained using a classical wave theory of light). One such experiment was performed by Grangier and coworkers [8], and it is this experiment that we have adapted. The basic idea is to show that if a single photon is incident on a beam-splitter it can be detected at the transmitted port, or the reflected port, but not both—-there should be no simultaneously detected photons at the transmitted and reflected ports.

To generate the single photons we use spontaneous parametric down conversion. A crystal of beta-bariumborate (BBO) is pumped by the output of a $405 \mathrm{~nm}$ laser diode, producing signal and idler photon pairs centered at $810 \mathrm{~nm}$ via type-I down-conversion. The pairs go in separate directions: the idler photon goes to a detector $G$ and the signal photon goes to a non-polarizing beam-splitter. The detection of an idler photon at detector $G$ serves as a gate, which heralds the presence of a signal photon at the beam-splitter. This photon is then either transmitted to detector $T$, or reflected to detector $R$. Detector $G$ has a narrow (10 $\mathrm{nm}$ bandwidth, centered at $810 \mathrm{~nm})$ interference filter located in front of it, whereas in front of detectors $T$ and $R$ there are color glass filters (RG780) that transmit all wavelengths longer than $780 \mathrm{~nm}$.

Let $N_{G}$ be the number of detections at detector $G$ in the time interval $\Delta T, N_{G T}$ be the number of coincidence detections between detectors $G$ and $T$ ( $N_{G R}$ is defined similarly), and $N_{G T R}$ be the number of simultaneous threefold detections. It was proved by Grangier et al. [8] that a classical wave incident on the beam-splitter must satisfy the following inequality:

$$
g^{(2)}(0)=\frac{N_{G T R} N_{G}}{N_{G T} N_{G R}} \geq 1
$$

Here $g^{(2)}(0)$ is the conditional degree of second-order coherence of the field incident on the beam-splitter-it is conditioned on the presence of a photon being detected at the gate detector $[1,8]$. If one measures $g^{(2)}(0)<1$ the experiment is not explainable with classical waves and must be explained using a quantum mechanical field. We were able to measure $g^{(2)}(0)=0.0177 \pm 0.0026$; after accounting for accidental coincidences this result is consistent with a single photon being incident on the beam-splitter.

Once students have shown that they can generate a field containing a single photon, the next obvious experiment is to show that a single photon will interfere with itself after traversing an interferometer. To do this we replace the beam-splitter with a polarization interferometer [2]; a diagram of the interferometer is shown in Fig. 1.

Light enters the interferometer vertically polarized. It then passes through a half-wave plate (" $\lambda / 2$ " in Fig. 1) that rotates the polarization of the beam to have both vertical and horizontal components. The beam then encounters a beam displacing prism (BDP), which is a piece of calcite cut so that the extraordinary (horizontally polarized) wave is displaced from the ordinary (vertically polarized) wave as it propagates. These beams will have equal amplitudes if the first half-wave plate is adjusted so that the light is polarized at $45^{\circ}$ as it enters the BDP. The half-wave plate after the BDP is oriented such that it flips the polarizations of the two beams so that their behavior is reversed at the second BDP. This is a necessary step in order to equalize the path lengths between the two arms, as described

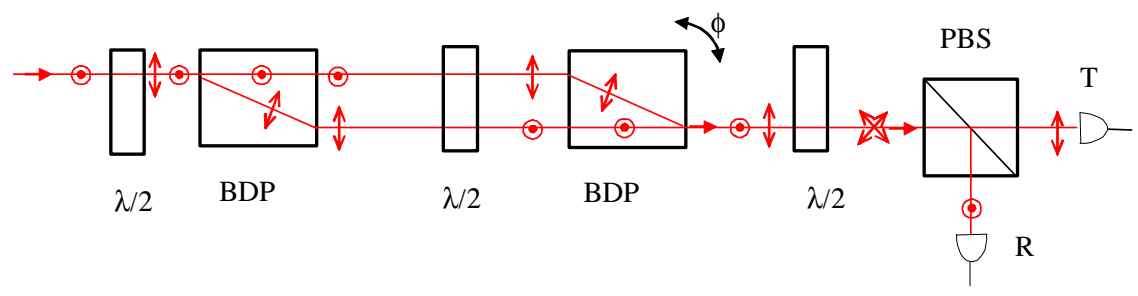

Fig. 1 The polarization interferometer with the polarizations of the beams indicated. 
further below. The second BDP is oriented to recombine the two beams, but since they are orthogonally polarized at this point they do not yet interfere. These orthogonally polarized beams then pass through another $\lambda / 2$ plate which rotates the two polarizations by $45^{\circ}$. The beams then hit a polarizing beam-splitter (PBS) where they interfere. The interference can be observed by tilting one of BDP's to sweep the relative phase.

This interferometer design has several advantages. Most importantly it can be made extremely compact, and it is made entirely from transmissive optical elements; this means that it is extremely stable, which allows us to use long counting times to obtain accurate statistics. Also, since the individual photons have large bandwidths the lengths of the two arms must be matched to a high precision in order to observe interference; this is easily achieved in this interferometer by simply rotating one of the BDP's.

Interference fringes can be seen in Fig. 2. Figure 2(a) shows the singles counts on detector $T, N_{T}$. This simply shows the classical "white-light" fringes of our broadband down-conversion source. Figure 2(b) shows the GT coincidence counts; since the detections at $T$ are now conditioned by a gate detection these represent events in which a single photon has traversed both paths of the beam-splitter and interfered with itself. The visibility of this interference pattern is $89 \%$. Lastly, since we are able to measure both output ports of the interferometer, we have all of the information necessary to simultaneously measure $g^{(2)}(0)$ with the interference fringes. Equation (1) is still valid for $g^{(2)}(0)$ as we scan the path-length difference because $g^{(2)}(0)$ is independent of the splitting ratio of the beam-splitter; an interferometer may be thought of as a beam-splitter with a splitting ratio that depends on the pathlength difference. In Fig. 2(c) we see that $g^{(2)}(0)<1$ at all times, verifying that we have only one photon in the interferometer at a given time. Thus, in this experiment we are able to simultaneously observe both the wave-like [Fig. 2(b)] and the particle-like [Fig. 2(c)] natures of light. We note that this result is not new, having been obtained before by Kwiat and Chiao [9]. We have simplified the experiment, however, and in doing so have made it possible for undergraduates to perform it as part of a teaching laboratory.

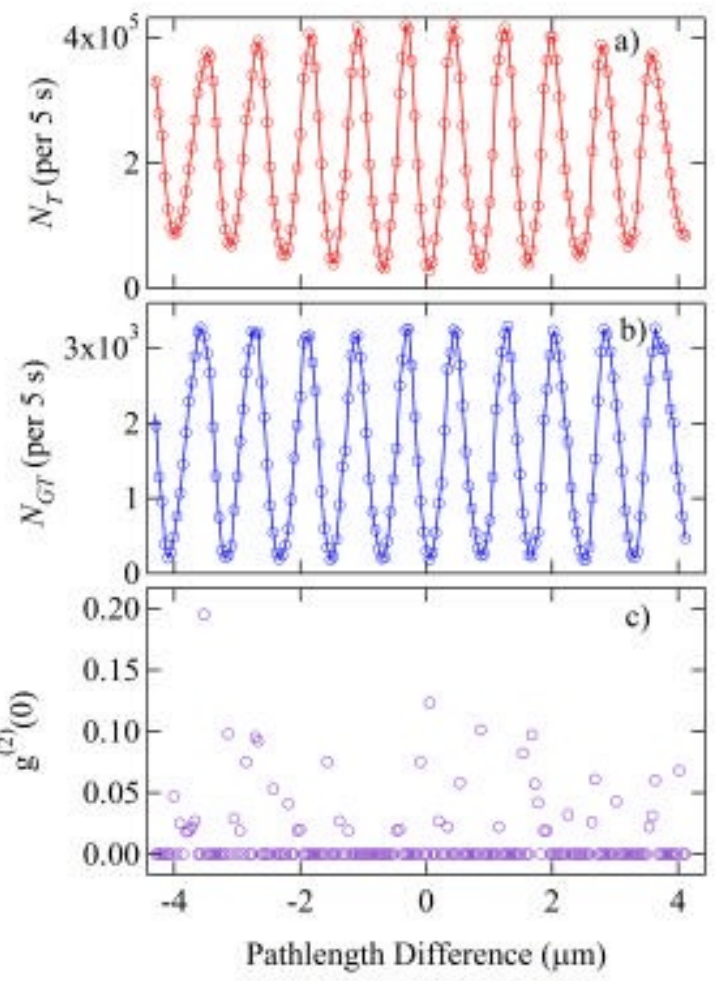

Fig. 2 a) shows measured singles counts on detector $T$, b) shows $G T$ coincidence counts, and c) shows the measured value of $g^{(2)}(0)$. All data are plotted as a function of the path-length difference between the two arms of the interferometer. 


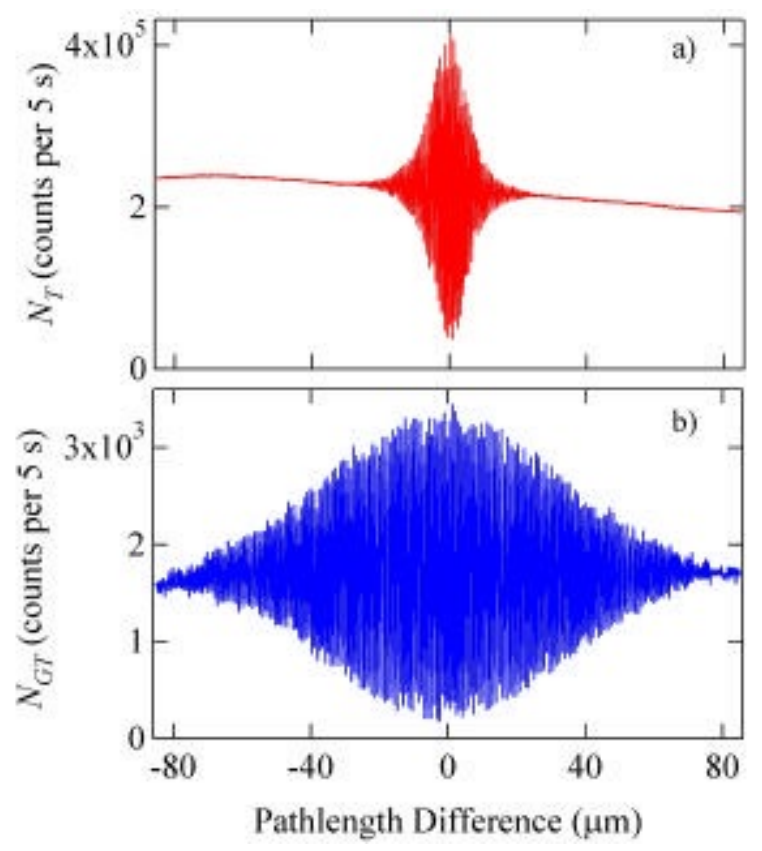

Fig. 3 a) shows measured singles counts on detector $T$, and b) shows $G T$ coincidence counts. All data are plotted as a function of the path-length difference between the two arms of the interferometer.

It can be seen in Fig 2(a) that the visibility of the fringe pattern is decreasing at larger pathlength differences, while this is not apparent in Fig. 2(b). Figure 3 shows longer scans which more clearly display the visibility envelopes. Here it is clearly seen that the fringe pattern measured in coincidence (the single photon data) has a longer coherence time (narrower bandwidth) than the singles counts. Why is this? The coherence length of the unconditioned downconversion in Fig. 2(a) is approximately 12 microns, which corresponds to a bandwidth of roughly $57 \mathrm{~nm}$; this is consistent with the bandwidth of the downconverted photons that we expect to collect. The coherence length of the conditioned downconversion in Fig. 2(b) is approximately 79 microns, which corresponds to a bandwidth of roughly $8.6 \mathrm{~nm}$; this is close the bandwidth of the interference filter in front of detector $\mathrm{G}$.

Thus, narrowing the bandwidth of the gating photons increases the coherence length of the interference pattern measured in coincidence. This is because the frequencies of the signal and idler are correlated - they are constrained by the fact that the sum of the signal and idler frequencies must be equal to the frequency of the pump photon. Indeed, the quantum theory of down-conversion indicates that the frequencies are entangled, but this experiment proves only that they are correlated. This effect was also seen in Refs. [9] and [5].

\section{Two Photon Interference}

Single-photon interference is a good starting point for studying quantum interference. Consider the Mach-Zehnder interferometer shown in Fig. 4. There are several ways in which one can use quantum mechanics to understand the interference of single photons going through the interferometer. One could use Feynman's approach of probability amplitudes for indistinguishable paths [10], state vector transformations by the interferometer elements [5], matrix operations of interferometer transformations [4], or even photon number state transformations [11].

In contrast to classical-wave interference, where interference depends on the number of paths, in quantum mechanics we consider the indistinguishable ways in which we can obtain a certain result. Two-photon interference provides such a distinction, because two photons traveling through the two possible paths of an interferometer interfere in four possible ways [5]. Thus, this experiment underscores the quantum mechanical view of interference. As described in Ref. [5], when all four possibilities are indistinguishable the probability for detecting the 


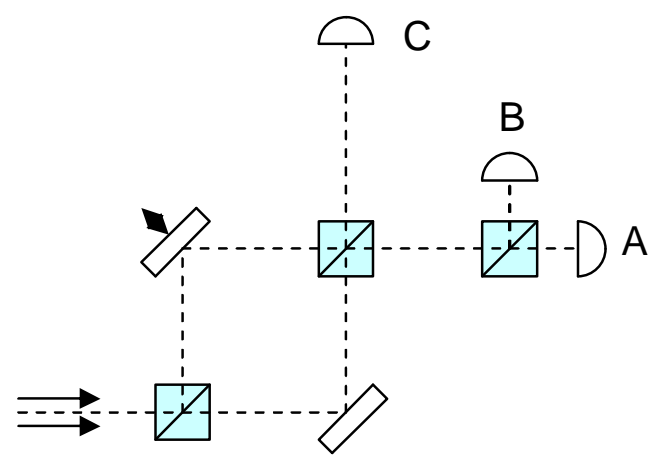

Fig. 4 Schematic of a Mach-Zehnder interferometer for two photons.

coincidences is

$$
P=1 / 4(1+\cos \delta)^{2}
$$

where $\delta$ is the phase difference due to the path-length difference of the interferometer arms. This is in contrast to single-photon interference, where the probability of the photon going through the interferometer is

$$
P=1 / 2(1+\cos \delta) .
$$

In this section we expand on the two photon interference problem by considering the case where the two photons enter the Mach-Zehnder interferometer together but leave it separately. We will use the state vector appro ach to describe the experiment. Let's represent the state of the light moving in the $x$ and $y$ directions by $|x\rangle$ and $|y\rangle$, respectively. In going through the a beam splitter these states transform as

$$
|x\rangle \rightarrow t|x\rangle+r|y\rangle
$$

and

$$
|y\rangle \rightarrow r|x\rangle+t|y\rangle
$$

where $t=1 / \sqrt{2}$ and $r=i / \sqrt{2}$ are the probability amplitudes for transmission and reflection at the beam-splitter, respectively. The state of the two identical photons before they enter the interferometer is given by the product state $|x\rangle_{1}|x\rangle_{2}$. In going through the interferometer the state of the light becomes

$$
|x\rangle_{1}|x\rangle_{2} \rightarrow r^{2} t^{2}\left(1+e^{i \delta}\right)^{2}|x\rangle_{1}|x\rangle_{2}+\left(r^{2}+t^{2} e^{i \delta}\right)^{2}|y\rangle_{1}|y\rangle_{2}+r t\left(1+e^{i \delta}\right)\left(r^{2}+t^{2} e^{i \delta}\right)\left(|x\rangle_{1}|y\rangle_{2}+|y\rangle_{1}|x\rangle_{2}\right) .
$$

The first term corresponds to the case already investigated in Ref. [5]. The rate of coincidences in detectors $A$ and $B$ is proportional to the probability of ending in state $|x\rangle_{1}|x\rangle_{2}$, which is given by Eq. (2). The probability of ending in state $|y\rangle_{1}|y\rangle_{2}$ differs from the one given by Eq. (2) in that it has a minus sign instead of a plus sign. Here we are interested in the case where the photons come out from the separate ports of the interferometer. It is interesting that the algebra leading to the state of the light coming from separate ports gives the symmetric wavefunction

$$
|\psi\rangle_{x y}=\frac{1}{\sqrt{2}}\left(|x\rangle_{1}|y\rangle_{2}+|y\rangle_{1}|x\rangle_{2}\right) .
$$


This is of course what we expect because photons are bosons! The probability for ending in this state $|\psi\rangle_{x y}$ is

$$
P=1 / 4(1-\cos 2 \delta) \text {. }
$$

This is a very interesting result. The probability oscillates with twice the frequency of the single-photon result. Notice that the visibility for this case can be 1 . This type of quantum interference has received much attention for improving over the classical limit of resolution [12]. This interference is similar to the one that gives rise to the Hong-Ou-Mandel interference [13], which produces a characteristic "dip." The dip disappears when the path length exceeds the coherence length of the down-converted light. In this case the visibility of the interference pattern will go to zero.

The picture of the experimental arrangement is shown in Fig. 5. Light from a GaN diode laser $(402 \mathrm{~nm})$ is sent through a BBO crystal producing photon pairs via type-I parametric down conversion. We orient the crystal for collinear down conversion of the pairs with equal energy $(804 \mathrm{~nm})$ [5]. A polarizer after the crystal eliminates the pump beam, which has a polarization orthogonal to that of the down-converted photons. The photon pairs enter a Mach-Zehnder interferometer. One of the mirrors of the interferometer is mounted on a linear stage that has a piezoelectric crystal as a spacer between the micrometer and the stage. After the interferometer the light is directed to three optical fiber couplers preceded by irises and 10-nm bandpass filters. The fibers are multimode and send the light to high efficiency single-photon avalanche photodiode detectors. More details of the apparatus, alignment, and cost can be found in our websites [14,15].

Figure 6 shows three data sets. The "+" symbols show the single-photon interference signal from detector $A$ only. In order to display the data with the other two data sets we plot $\left(N_{A} / 80+1500\right)$. The data set represented by the symbols " $x$ " shows the coincidences between detectors $A$ and $B, N_{A B}$, displaying the type of interference represented by Eq. (2). The third set of symbols (filled circles) show the coincidences between detectors $A$ and $C, N_{A C}$. Notice that the frequency of the interference is twice that of the single-photon interference, in agreement with Eq. (8). The apparent phase shift between the data sets is due to the placement of the irises after the interferometer.

This setup is harder to align than, for example, the setup of Sec. 1. The arms need to be aligned to near zero pathlength difference. We have developed a method for systematically aligning the interferometer [15]. Once the interferometer is aligned then search for white-light fringes by putting a small incandescent bulb at the entrance of the interferometer and placing at the output a multimode fiber that sends the light to a low-resolution spectrometer. We have used this setup for the laboratory of the quantum mechanics course at Colgate University. For more details see our website [15].

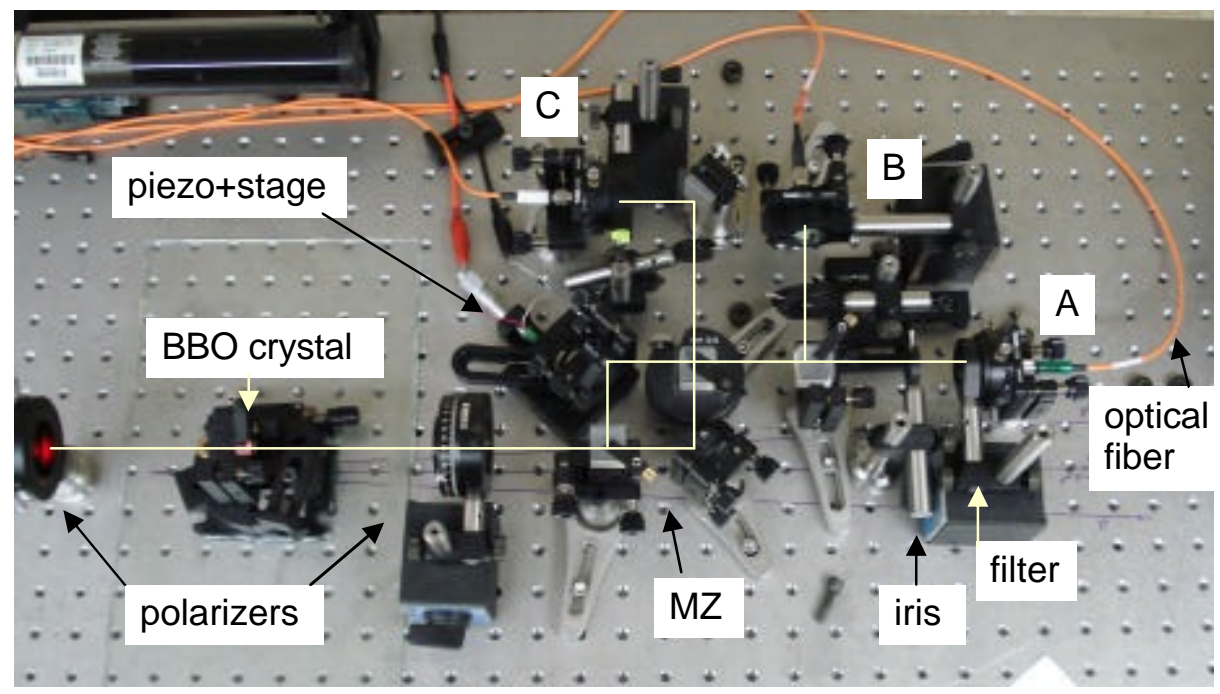

Fig. 5. Photo of the apparatus used for the two photon experiments. 


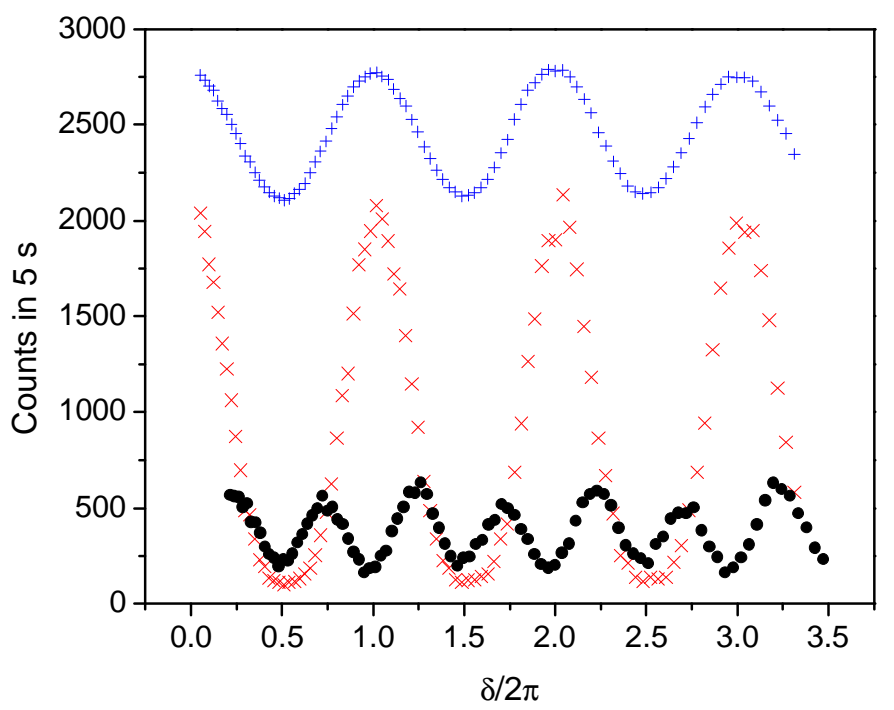

Fig. 6. Graph of the results of the two-photon interference experiment. Symbols (+) are proportional to the single-photon counts $N_{A}$; (x) represent the counts when both photons leave the interferometer through the same port $N_{A B}$; and $(\bullet)$ represent the counts when the photons leave the separate arms of the interferometer $N_{A C}$.

\section{Conclusions}

In conclusion, we present new results from one and two-photon interference experiments that underscore the quantum nature of light. The experiments are a useful laboratory aid for teaching quantum mechanics and quantum optics. These programs have been successfully implemented at our undergraduate colleges. We foresee that the experiments could also be implemented in graduate-level courses in quantum optics.

\section{Acknowledgments}

We'd like to acknowledge the contributions of R. E. Davies, V. W. Donato, A. Gogo, C.H. Holbrow, W. D. Snyder and G. S. Bergreen. This work was supported by grants DUE-0442882 and DUE-0340917 from the National Science Foundation and by internal funds from Colgate University and Whitman College.

\section{References}

[1] J. J. Thorn, M. S. Neal, V. W. Donato, G. S. Bergreen, R. E. Davies, and M. Beck, "Observing the quantum behavior of light in an undergraduate laboratory," Am. J. Phys. 72, 1210-1219 (2004)

[2] A. Gogo, W.D. Snyder, and M. Beck, “Comparing quantum and classical correlations in a quantum eraser,” Phys. Rev. A 71, 052103 (2005)

[3] J.A. Carlson, D.M. Olmstead, and M. Beck, “Quantum mysteries tested: An experiment implementing Hardy's test of local realism,” Am. J. Phys. 74, 180-186 (2006)

[4] C.H. Holbrow, E.J. Galvez, and M.E. Parks, "Photon quantum mechanics and beam splitters," Am. J. Phys. 70, $898-902$ (2002).

[5] E. J. Galvez, C. H. Holbrow, M. J. Pysher, J. W. Martin, N. Courtemanche, L. Heilig, and J. Spencer, "Interference with correlated photons: Five quantum mechanics experiments for undergraduates," Am. J. Phys. 73, 127-140 (2005).

[6] D. Dehlinger and M. W. Mitchell, "Entangled photon apparatus for the undergraduate laboratory," Am. J. Phys. 70, 898-902 (2002).

[7] D. Dehlinger and M. W. Mitchell, "Entangled photons, nonlocality, and Bell inequalities in the undergraduate laboratory," Am. J. Phys. 70, 903-910 (2002).

[8] P. Grangier, G. Roger, and A. Aspect, "Experimental evidence for a photon anticorrelation effect on a beam splitter: A new light on singlephoton interferences," Europhys. Lett. 1, 173-179 (1986).

[9] P. G. Kwiat and R. Y. Chiao, "Observation of a nonclassical Berry phase for the photon," Phys. Rev. Lett. 66, 588-591 (1991).

[10] R.P. Feynman, R.B. Leighton, and M. Sands, The Feynman Lectures on Physics (Addison-Wesley, 1965), Vol. 3, p. 1-1.

[11] C.C. Gerry and P.L. Knight, Introductory Quantum Optics (Cambridge University Press, Cambridge, 2005).

[12] A.N. Boto, P. Kok, D.S. Abrams, S.L. Braunstein, C.P. Williams, and J.P. Dowling, "Quantum interferometric optical lithography: To beat the diffraction limit," Phys. Rev. Lett. 85, 2733-2736 (2000).

[13] C.K. Hong, Z.Y. Ou, and L. Mandel, "Measurement of subpicosecond time intervals between two photons by interference,” Phys. Rev. Lett. 59, 2044-2046 (1987).

[14] http://marcus.whitman.edu/ beckmk/QM.

[15] http://departments.colgate.edu/physics/pql.htm. 